\title{
Los Mapas Conceptuales y el Aprendizaje Basado en Proble- mas en el Aprendizaje de Contenidos Anatómico-fisiológicos en Opositores al Cuerpo de Profesores de Educación Secundaria
}

\author{
Concept Mapping and Problem-Based Learning Content in Anatomical and Physiological \\ in Students for a Public Competition To Be Teachers in Secundary Education
}

Antonio Baena-Extremera \& Antonio Granero-Gallegos

BAENA-EXTREMERA, A. \& GRANERO-GALLEGOS, A. Los mapas conceptuales y el aprendizaje basado en problemas en el aprendizaje de contenidos anatómico-fisiológicos en opositores al cuerpo de profesores de Educación Secundaria. Int. J. Morphol., 30(1):230-237, 2012.

RESUMEN: El objetivo de este trabajo ha sido comparar el rendimiento en el aprendizaje de alumnos que se prepararon la oposición para el acceso el cuerpo de profesorado de secundaria en la especialidad de Educación Física, tras recibir la formación de distintos bloques temáticos de anatomía y fisiología a través de diferentes métodos en enseñanza-aprendizaje: mapas conceptuales (CMaps), aprendizaje basado en problemas (ABP) y utilización combinada de ambos métodos. Metodología: Se utilizó un diseño cuasiexperimental, descriptivo y seccional. La muestra total se compuso por 180 estudiantes, 95 varones y 85 mujeres. Se trabajó con un grupo de 64 personas que recibieron formación a través de CMaps, otro de 61 alumnos que recibió formación a través de ABP, y un tercer grupo de 55 que trabajaron combinando ambos métodos. El instrumento de medida fue un cuestionario de evaluación tipo test para cada bloque de contenidos. Se obtuvo una medida pretest antes de la formación y otra medida después de la intervención (postest). Se han analizado las mejoras de cada grupo según el método de enseñanza aplicado y se han comparado los resultados obtenidos mediante análisis de la puntuación de ganancia (postest-pretest) con T de Student para muestras relacionadas. Tras la intervención la media de respuestas correctas es superior al ochenta por ciento en los cinco bloques analizados, siendo los de fisiología y anatomía en los que más se evolucionó. El ABP ha resultado más efectivo que el CMaps, pero las mejoras más altas respecto al pretest corresponden al grupo que ha estudiado combinando CMaps+ABP. Los resultados se discutieron con otros autores, coincidiendo en la necesidad de plantearse una mayor utilización del ABP como método de enseñanza-aprendizaje y que mediante un enfoque metodológico mixto el alumnado adquiere mayores habilidades y competencias.

PALABRAS CLAVE: Metodología; Mapas conceptuales; Aprendizaje basado en problemas.

\section{INTRODUCCIÓN}

En España, el proceso para poder llegar a ser docente funcionario del estado en el cuerpo de profesorado de educación secundaria consiste en aprobar un exigente y riguroso concurso-oposición convocado, a veces, a nivel estatal. Una de las pruebas más determinantes es la elaboración de forma escrita, durante dos horas continuadas, de un tema elegido por sorteo del temario correspondiente a la especialidad educativa a la que se aspire. En el caso del área de Educación Física el temario actual está compuesto por 65 temas (Orden de 9 de septiembre de 1993 por la que se aprueba los temarios que han de regir en los procedimientos selectivos del Cuerpo de Profesores, BOE de 21-sep-1993), dentro de los cuales destacamos cinco bloques dedicados a Anatomía, Fisiología, Salud Corporal, Capacidades Físicas
Básicas y Condición Física Orgánica. Desde hace años, estos bloques han sido los que más protagonismo han tenido dentro de los estudios de la Licenciatura en Ciencias de la Actividad Física y del Deporte y del proceso de concursooposición debido a su gran dificultad en el aprendizaje.

Por lo general, la metodología utilizada para la enseñanza-aprendizaje de estos bloques de temas suele ser tradicional y mediante clase magistral, en la que el docente explica detalladamente el temario y el alumno adquiere un papel de mero participante pasivo y receptor de información. Ante el uso de la metodología tradicional, y para reducir al máximo la información, algunos profesores comienzan a trabajar para el estudio de los bloques especificados otros métodos y 
técnicas más innovadores y constructivistas, como son los mapas conceptuales (CMaps) y el aprendizaje basado en problemas (ABP), aún sin tener absoluta certeza de si son más o menos eficaces para el aprendizaje de los discentes.

Según Novak \& Gowin (1984) y Novak (1998), los CMaps representan una colección de conceptos interconectados con relaciones específicas entre ellos. Lo ideal en la creación de CMaps es seguir la estructura del conocimiento y no al revés, consiguiendo incluso que el aprendizaje sea significativo. Existen investigaciones realizadas con CMaps en relación al aprendizaje de ciertas materias. Por ejemplo, Soyibo (1995) comprobó que es adecuado usar CMaps para evaluar diferencias en relación a temas de respiración presentados en diferentes libros. Edmonson (1995) examinó el efecto positivo del uso de CMaps en el desarrollo de un currículo basado en problemas veterinarios; o el trabajo de Flórez-Uribe et al. (2011), quienes sugieren la utilización de los CMaps de forma cooperativa para mejorar el proceso de enseñanza y aprendizaje en asignaturas como la neuroanatomía.

Otra metodología utilizada cada vez más en la enseñanza universitaria es el aprendizaje basado en problemas (ABP) o Problem-Based Learning. "En este enfoque se privilegia el auto-aprendizaje y la auto-formación, procesos que son facilitados por la dinámica del enfoque y la concepción constructivista ecléctica del mismo" (Dueñas, 2001). Numerosas facultades de medicina de todo el mundo están utilizando estos enfoques pedagógicos y metodológicos (Dueñas), como en Toronto o Western (Newman, 1993) o en Chile (Friedman et al., 1990), entre otros. Por ello, la utilización de situaciones-problema, como en los casos clínicos, son comunes en las diferentes facultades de ciencias de la salud para desarrollar tópicos distintos (Canalejas et al., 2005; Williams, 2005), pudiendo ser ideales también para estudiar los temas relacionados con los bloques de contenidos anteriormente descritos.

A pesar de las bondades de ambos enfoques pedagógicos, otra de las posibilidades sería utilizar una combinación de ambos, de esta forma se mejoraría el autoaprendizaje significativo, el pensamiento crítico y a la evaluación formativa, cualitativa e individualizada (Rendas et al., 2006). Un ejemplo lo podemos encontrar en Ayala-Pimentel et al. (2003), quienes utilizan una metodología que combina los CMaps con el ABP en la enseñanza de la neuroanatomía, consiguiendo resultados muy satisfactorios.

Para enseñar y que los alumnos aprendan eficazmente los temas relacionados con los bloques indicados anteriormente para el acceso al cuerpo de funcionarios docentes, en las academias de preparación cada profesor sigue un enfo- que metodológico diferente. En cambio, aún no se ha determinado que método de enseñanza sería el más adecuado para trabajar aquellos temas relacionados con los bloques especificados. Por ello, el objetivo de esta investigación fue comparar el rendimiento en el aprendizaje de alumnos y alumnas que se prepararon la oposición para el acceso el cuerpo de profesorado de secundaria en la especialidad de Educación Física, tras recibir la formación en Anatomía, Fisiología, Salud Corporal, Cualidades Físicas Básicas y Condición Física Orgánica a través de diferentes métodos en enseñanza-aprendizaje: CMaps, ABP y utilización combinada de ambos métodos.

\section{MATERIAL Y MÉTODO}

Muestra. La muestra se compuso de 180 alumnos con edades comprendidas entre los 24 y 29 años, con un total de 95 varones $(M=26,02 ; \mathrm{DT}=, 91)$ y 85 mujeres $(\mathrm{M}=25,34$; $\mathrm{DT}=, 81)$. Todos ellos son titulados con la Licenciatura en Ciencias de la Actividad Física y del Deporte que prepararon oposiciones para el acceso al cuerpo de Profesores de Educación Secundaria en España durante el curso 2009/2010.

Diseño. Se utilizó un diseño cuasi-experimental, descriptivo y seccional. Se trabajó con un grupo de 64 personas, divididos en dos subgrupos mixtos de 31 y 33 personas, que recibieron la formación a través de CMaps (Grupo A). Otro grupo de 61 personas, divididos en otros dos subgrupos mixtos de 29 y 32, recibieron la formación teórica del temario especificado a través del ABP (Grupo B). Y un tercer grupo de 55 alumnos, dividido en dos subgrupos de 23 y 22 , recibieron sus clases utilizando la combinación de ambos métodos para el mismo bloque de temas (Grupo C). Todas las clases tuvieron la misma duración para todos los grupos y se trataron los mismos contenidos, pero diferenciando los enfoques metodológicos indicados.

Se realizó una primera medida a todos los grupos (pretest) para conocer el nivel inicial de conocimientos. Esta medida pretest se realizó antes del inicio de cada bloque temático. Este pretest se utilizó también para evaluar la normalidad de la muestra, siendo desestimadas 3 personas en el grupo A, 3 en el grupo B y 4 en el grupo C, por demostrar conocimientos fuera de la normalidad. Posteriormente se realizó otra medida (postest) a todos los grupos cada vez que se finalizaba el proceso enseñanza-aprendizaje de cada bloque temático, para establecer comparaciones con el pretest.

Hipótesis. Las hipótesis planteadas en esta investigación fueron: 
1.- Se cree que a partir de la intervención se producirán diferencias significativas de medias de respuestas correctas entre pretest y postest (diferencias intragrupos) en cada uno de los bloques temáticos.

2.- Se piensa que el mayor incremento de aprendizaje se producirá en aquel alumnado que ha recibido las clases combinando los dos métodos de enseñanza. Asimismo, se cree que el ABP produce mayores mejoras de aprendizaje que los CMaps.

Instrumento de medida. Para llevar a cabo las evaluaciones de los conocimientos se diseñó un cuestionario para cada bloque compuesto por 50 preguntas tipo test, de selección múltiple y con una sola respuesta válida. Todos los exámenes se prepararon antes de la intervención, por el equipo de profesorado e investigadores, con el fin de evitar cualquier tipo de sesgo en la preparación de las pruebas tras la intervención. En este instrumento se hizo referencia a la mayor parte de los contenidos de cada bloque y se abarcaron todos los temas en su máxima amplitud posible. En los ejercicios de pretest se dejó un tiempo de 90 minutos para responder a las cuestiones. En los postets, el tiempo máximo fue de 60 minutos.
Bloques temáticos. La formación teórica estaba dividida en cinco bloques compuestos por diferentes temas que se detallan en la Figura 1.

Procedimiento. Cada grupo recibió las sesiones en el aula por un profesor experto en Anatomía y Fisiología del Ejercicio, con amplios conocimientos en didáctica y en la utilización de los CMaps y ABP. Dicho profesor, que contribuyó en el diseño de la intervención y contó en todo momento con el asesoramiento del equipo de investigadores, posee más de cinco años de experiencia en la preparación de Licenciados en Educación Física y Ciencias de la Actividad Física y del Deporte para el concurso-oposición en los bloques especificados.

El proceso de formación de esta investigación se realizó durante el curso escolar 2009/2010, en sesiones celebradas los martes, miércoles y jueves desde febrero a junio, de 10:00 a 14:00 horas. Se utilizó un total de 4 horas por tema en cada uno de los bloques, tanto para la metodología de CMaps, ABP y combinadas. Todos los grupos recibieron las clases por el mismo profesor.

Anatomia (Temas 9,21 y 27 ). Contenidos
a tratar:
- Estructura del ejercicio físico.
- Forma y técnica.
- Análisis de los elementos mecánicos,
kinesiológicos y funcionales del cuerpo.
- El sistema óseo-articular.
- Estructura y funciones del sistema óseo-
articular.
- Características particulares del sistema
óseo-articular del periodo evolutivo
correspondiente de los 12 a los 18 años.
- El sistema cardio-respiratorio.
- Estructura y funciones del sistema
cardio-respiratorio.
- Características particulares del sistema
cardio-respiratorio del periodo evolutivo
correspondiente de los 12 a los 18 años.

Capacidades Físicas Básicas (Temas

$14,20,22,24,26$ ). Contenidos a tratar.

- Principios básicos para el adecuado

desarrollo de la condición física.

- La resistencia y sus tipos.

- La fuerzay sus tipos.

- Lavelocidady sus tipos.

- La flexibilidad y sus tipos.

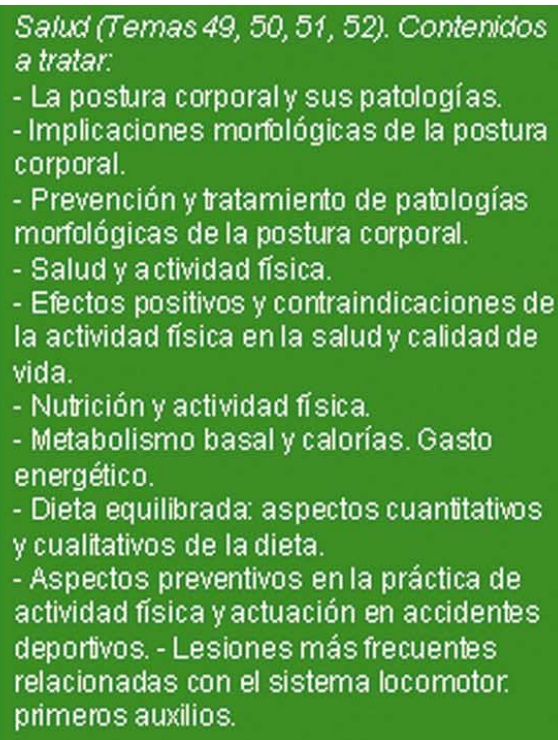

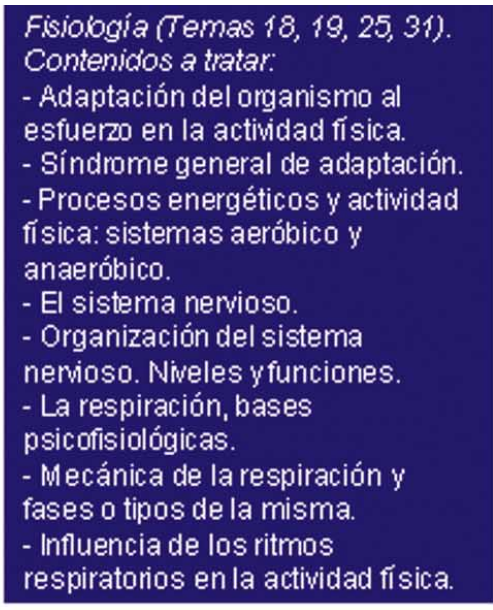

Fisiologia (Temas 18, 19, 25, 31).

Contenidos a tratar.

- Adaptación del organismo a

onico.

- La respiración, bases

psicofisiológicas.

- Mecánica de la respiración y

ases o tipos de la misma.

respiratorios en la actividad física.

Fig. 1. Bloques temáticos y contenidos.

Condición Fisica Orgánica (Temas 15, 17). Contenidos a tratar:

- El calentamiento: fundamentos y tipos.

- Funciones del calentamiento y rendimiento.

- Calentamiento y prevención de lesiones.

- Criterios para la elaboración de tareas de calentamiento general y

especííco.

- Factores que intervienen en el trabajo de desarrollo de la condición

física: intensidady volumen; recuperación, duración y repeticiones. 
La medida pretest se realizó en la primera clase teórica, antes de comenzar con la formación; mientras que los postests se realizaron una semana después de terminar cada bloque con la metodología utilizada. De esta manera, corregimos las limitaciones aportadas por Alleyne et al. (2002) y Jones et al. (2002), en estudios de este tipo.

Análisis. Para el análisis estadístico, las respuestas correctas se codificaron con el número 1 y las incorrectas con el 0. En cada uno de los bloques se calcularon los estadísticos descriptivos, media (M) y desviación típica (DT). Se realizaron las pertinentes pruebas de normalidad y homocedasticidad para asegurar la homogeneidad de la varianza. Se han calculado los índices de asimetría y curtosis siendo, en general, próximos a cero y $<2$, tal y como recomiendan Bollen \& Long (1994). De forma preliminar se realizó el cómputo de respuestas correctas de cada grupo y en cada uno de los bloques temáticos. Una vez desarrollado el programa de intervención, para estudiar las diferencias intragrupo en las diferentes tomas (pre y postest) se ha realizado un análisis de las puntuaciones de diferencia o análisis de puntuación de ganancia (Best, 1970). Para ello se ha calculado la diferencia entre las puntuaciones pretest y postest para cada persona, analizando a continuación las diferencias intragrupo mediante la prueba T de Student para muestras relacionadas. Para todo ello se empleó el paquete estadístico SPSS en su versión 17.0 para Windows.

\section{RESULTADOS}

Análisis preliminar. En primer lugar, y de forma previa a la intervención, se realizó una medida pretest para comprobar el punto de partida de los distintos participantes en el programa. En la Tabla I se puede comprobar la media de respuestas correctas en cada uno de los bloques temáticos, sobre las 50 cuestiones propuestas. Se observa que en ninguno de los casos la media alcanza el cincuenta por ciento de respuestas correctas, por lo que no superarían las pruebas tipo test. Los bloques temáticos en los que se obtiene mejor puntuación son los de condición física orgánica $(\mathrm{M}=22,74)$ y capacidades físicas básicas $(M=22,43)$. En el bloque de salud desciende la media hasta 18,63. Los peores resultados iniciales corresponden a anatomía $(M=15,73)$ y fisiología, en el que apenas se logra una media de 12,46 respuestas correctas.

Efectos de la intervención. Tras la intervención se realizó una medida postest en cada uno de los bloques temáticos. En la Tabla I se puede apreciar la evolución general del total de la muestra en los diferentes bloques. Después del programa la media de respuestas correctas es superior al ochenta por ciento enlos cinco bloques, confirmándose así la primera hipótesis del estudio. Los mejores resultados los presentan condición física orgánica $(M=42,23)$, capacidades físicas básicas $(M=41,61)$ y anatomía $(M=41,59)$, aunque los resultados de fisiología y salud también superan las 40 respuestas correctas.

Los contenidos en los que más han evolucionado los alumnos son los de fisiología y anatomía, dado que en ellos había más errores en la medida pretest. La puntuación de ganancia (postest-pretest) es mayor en estos temas indicados. No obstante, para conocer qué método de enseñanza-aprendizaje ha resultado más efectivo, se ha estudiado la puntuación de ganancia de cada uno de los grupos (A, B y C) mediante la Prueba T de Student para muestras relacionadas (diferencias intragrupo). En la Tabla II se pueden observar la M, DT y puntuación de ganancia de cada uno de los grupos, tanto en el pretest como en el postest.

Tabla I. Estadísticos descriptivos de la totalidad de la muestra. Medidas pretest y postest en cada bloque temático.

\begin{tabular}{llcccccc}
\hline Bloques temáticos & & $\boldsymbol{M}$ & Mínimo & Máximo & DT & Asimetría & Curtosis \\
\hline \multirow{2}{*}{ Anatomía } & Pretest & 15.73 & 13 & 18 & 1.21 & .21 & -.44 \\
& Postest & 41.59 & 36 & 48 & 4.11 & .23 & -1.33 \\
Fisiología & Pretest & 12.46 & 10 & 15 & 1.15 & -.39 & -.12 \\
& Postest & 40.50 & 35 & 48 & 3.75 & .33 & -.91 \\
Salud Corporal & Pretest & 18.63 & 16 & 22 & 1.07 & .39 & -.04 \\
& Postest & 40.76 & 37 & 46 & 2.19 & .42 & -.70 \\
Cualidades Física Básicas & Pretest & 22.43 & 20 & 26 & 1.04 & .57 & .58 \\
& Postest & 41.61 & 36 & 46 & 3.14 & -.27 & -1.43 \\
Condición Física Orgánica & Pretest & 22.74 & 19 & 26 & 1.51 & -.47 & .67 \\
& Postest & 42.23 & 36 & 49 & 4.27 & .04 & -1.38 \\
\hline
\end{tabular}


BAENA-EXTREMERA, A. \& GRANERO-GALLEGOS, A. Los mapas conceptuales y el aprendizaje basado en problemas en el aprendizaje de contenidos anatómico-fisiológicos en opositores al cuerpo de profesores de Educación Secundaria. Int. J. Morphol., 30(1):230-237, 2012.

Tabla II. Media y desviación típica de pretest, postest y puntuación de ganancia (PG). Diferencias intra-grupos. Prueba T de Student para muestras relacionadas.

\begin{tabular}{|c|c|c|c|c|c|c|c|}
\hline \multirow[b]{2}{*}{ Bloques temáticos } & & \multicolumn{2}{|c|}{$\begin{array}{l}\text { Grupo A }(n=61) \\
\text { Método: CMaps }\end{array}$} & \multicolumn{2}{|c|}{$\begin{array}{c}\text { Grupo B }(n=58) \\
\text { Método: ABP }\end{array}$} & \multicolumn{2}{|c|}{$\begin{array}{c}\text { Grupo C }(n=51) \\
\text { Método: CMaps+ABP }\end{array}$} \\
\hline & & $M$ & $D T$ & $M$ & $D T$ & $M$ & $D T$ \\
\hline \multirow{5}{*}{ Anatomía } & Pretest & 15.25 & 1.09 & 16.43 & 1.23 & 15.51 & .95 \\
\hline & Postest & 36.79 & .80 & 41.33 & .94 & 46.67 & 1.01 \\
\hline & PG & 21.54 & & 24.9 & & 31.16 & \\
\hline & $t$ & -116.49 & & -119.55 & & -168.93 & \\
\hline & $p$ & .000 & & .000 & & .000 & \\
\hline \multirow{5}{*}{ Fisiología } & Pretest & 11.95 & 1.17 & 13.16 & .70 & 12.29 & 1.15 \\
\hline & Postest & 36.23 & .97 & 40.38 & 1.06 & 44.90 & 1.78 \\
\hline & PG & 24.28 & & 27.22 & & 32.61 & \\
\hline & $t$ & -129.69 & & -168.71 & & -108.54 & \\
\hline & $p$ & .000 & & .000 & & .000 & \\
\hline \multirow{5}{*}{ S alud Corporal } & Pretest & 19.25 & 1.13 & 17.97 & .70 & 18.67 & .89 \\
\hline & Postest & 38.56 & 1.01 & 40.57 & 1.11 & 43.14 & 1.41 \\
\hline & PG & 19.31 & & 22.6 & & 24.47 & \\
\hline & $t$ & -86.82 & & -114.04 & & -95.14 & \\
\hline & $p$ & .000 & & .000 & & .000 & \\
\hline \multirow{5}{*}{ Capacidades Físicas Básicas } & Pretest & 21.98 & .94 & 23.05 & 1.13 & 22.27 & .67 \\
\hline & Postest & 37.54 & .65 & 42.90 & 1.19 & 44.39 & 1.10 \\
\hline & PG & 15.56 & & 19.85 & & 22.12 & \\
\hline & $t$ & -93.63 & & -84.67 & & -140.39 & \\
\hline & $p$ & .000 & & .000 & & .000 & \\
\hline \multirow{5}{*}{ Condición Física Orgánica } & Pretest & 23.52 & 1.30 & 21.88 & 1.64 & 22.84 & .97 \\
\hline & Postest & 36.98 & .78 & 42.47 & .94 & 47.25 & .91 \\
\hline & PG & 13.46 & & 20.59 & & 24.41 & \\
\hline & $t$ & -89.29 & & -79.07 & & -117.35 & \\
\hline & $p$ & .000 & & .000 & & .000 & \\
\hline
\end{tabular}

Tras el proceso de estudio y aprendizaje se han producido mejoras significativas en todos los grupos y bloques de contenidos $(\mathrm{p} \leq, 000)$ (Tabla II). En los tres grupos las mayores diferencias intragrupo se han producido en los bloques de fisiología y anatomía, respectivamente. En el grupo A (CMaps): fisiología, $\mathrm{t}(60)=-129,69$ (p $\leq, 000)$ y anatomía, $\mathrm{t}(60)=-116,9(\mathrm{p} \leq, 000)$; en el grupo B (ABP): fisiología, $\mathrm{t}(57)=-168,79(\mathrm{p} \leq, 000)$ y anatomía, $\mathrm{t}(57)=-119,55(\mathrm{p} \leq, 000)$; en el grupo $\mathrm{C}(\mathrm{CMaps}+\mathrm{ABP})$ : fisiología, $\mathrm{t}(50)=-108,54$ $(\mathrm{p} \leq, 000)$ y anatomía, $\mathrm{t}(50)=-168,93(\mathrm{p} \leq, 000)$.

En la segunda hipótesis se pensó que el enfoque más efectivo sería el que utilizaba la combinación de ambos métodos, ABP y CMaps, así como que con el ABP se incrementaría más el aprendizaje que con los CMaps. Efectivamente, si se observan gráficamente las puntuaciones de ga- nancia de cada grupo en los diferentes bloques temáticos (Fig. 2), se comprueba que corresponden al grupo que ha estudiado con el método CMaps+ABP las mejoras más altas respecto al pretest, pues en todos los bloques se ha incrementado el número de respuestas correctas en más de veinte. Incluso, se ha alcanzado una puntuación de ganancia por encima de 30 en fisiología $(32,61)$ y anatomía $(31,16)$. Las mejoras en el grupo que ha estudiado mediante ABP también son destacables, con valores de ganancia en torno a 20 en salud corporal, capacidades físicas básicas y condición física orgánica, aunque resalta el bloque de fisiología, con una mejora de 27,22 y el de anatomía, con una mejora de 24,9. En el caso del grupo que ha estudiado mediante los CMaps, las mejoras son evidentes, aunque menores que en el resto de grupos. En este grupo destaca la puntuación de ganancia en fisiología y anatomía, con valores superiores a 20 (Tabla II). 


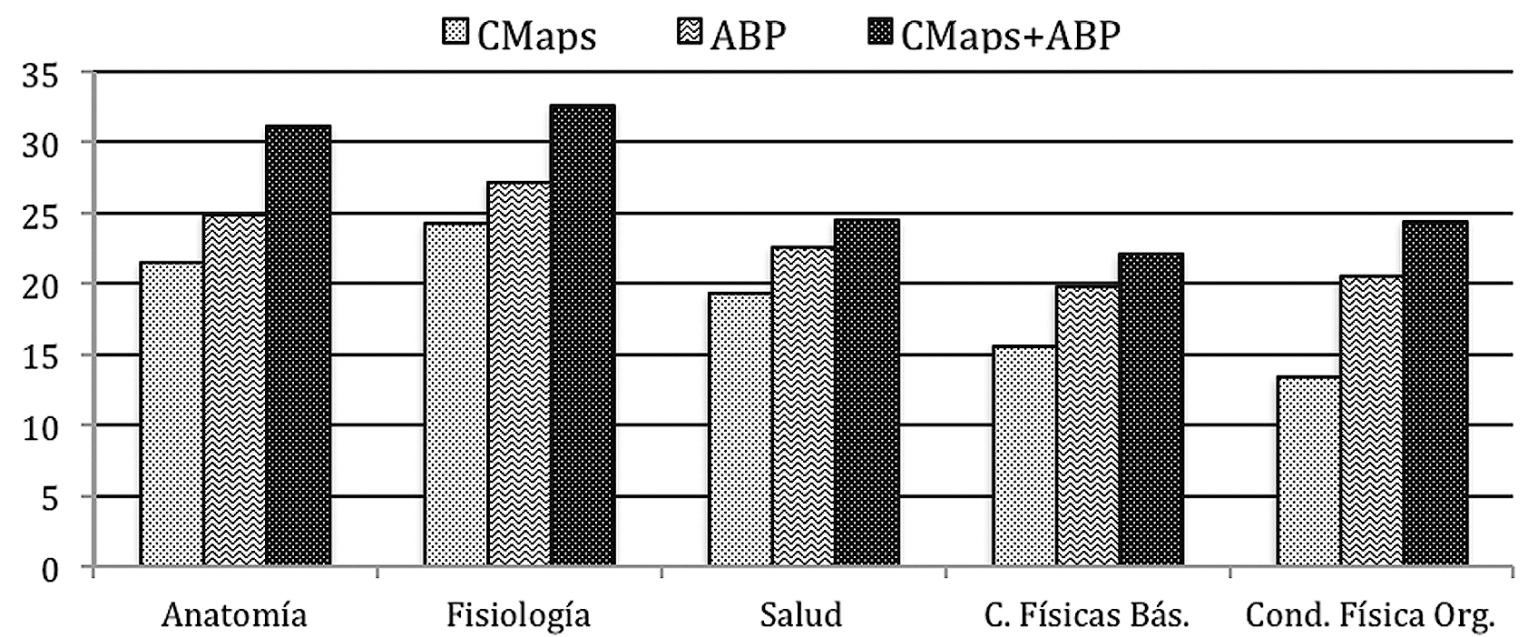

Fig. 2. Puntuaciones de ganancia en cada bloque temático según método de aprendizaje utilizado.

\section{DISCUSIÓN}

Teniendo como referente el objetivo de esta investigación, se ha podido comprobar la mejoría obtenida en todos los bloques con el uso de CMaps, aunque siempre algo menor que con el resto de métodos. Los resultados coinciden con los obtenidos por Flórez-Uribe et al., quienes encontraron diferencias significativas en el rendimiento de un grupo que trabajó con CMaps al final de la unidad de trabajo. Igualmente coincidimos con Hsu (2004), al comprobar cómo la utilización de los CMaps como estrategia de enseñanza constructivista e innovadora, permiten la obtención de resultados positivos.

Respecto al ABP, en la literatura se encuentran diversos estudios que se han realizado para evaluar los resultados de la aplicación de este método y comprobar sus bondades sobre los de enseñanza tradicional (Gómez et al., 2009; Patel et al., 1991) o, incluso, demostrar que no existen evidencias, como en el caso de estudiantes de medicina (Alleyne et al.). En esta investigación las mejoras han sido muy evidentes, incluso mayores que las obtenidas con el uso de los CMaps para cada bloque. Estos resultados indican que, en relación a los bloque temáticos tratados, mediante el ABP el alumnado aprende más que con el CMaps. Estos datos están en la línea de las aportaciones de Álvarez-Montero et al. (2001) en escenarios clínicos, donde pudieron comprobar la mejora en el rendimiento del aprendizaje de los alumnos. Se deduce, por tanto, que la utilización de metodologías en las que el alumno se implique en su aprendizaje, haciéndolo más constructivo a la vez que realiza prácticas innovadoras, ayudan a obtener mejores resultados en los procesos de enseñanza y aprendizaje, tal y como podemos observar en los resultados expuestos en este estudio, y que coinciden, ade- más, con Hsu y Michael (2001). En cambio, Smits et al. (2002), tras la revisión de seis estudios sobre ABP aplicado en la carrera de Medicina entre 1974 y 2000, no encontraron evidencias de que el ABP mejorara la adquisición de conocimientos de los discentes y su actuación.

Los mayores incrementos en el aprendizaje del alumnado se han producido al utilizar de forma combinada ambos métodos: CMaps con ABP. Estos resultados coinciden con los de Gal-Iglesias et al. (2009) sobre contenidos de Anatomía y Fisiología, pues se ha podido comprobar que con un enfoque metodológico mixto el alumnado adquiere mayores habilidades y competencias. Coincidimos, por tanto, con Ayala-Pimentel et al., en afirmar que es mejor utilizar una metodología mixta, ya que permite un trabajo colaborativo, y se produce mejora en el procesamiento de la información en los niveles superiores, tal como ocurre en la resolución de situaciones problemáticas.

A la vista de estos resultados, y coincidiendo con Dueñas y Gal-Iglesias et al., se deberían diseñar programas en los que se integren diferentes metodologías, en la proporción adecuada, para maximizar, tanto la adquisición de conocimientos como el desarrollo de competencias.

Como conclusión, decir que la mejor metodología utilizada es la combinación de ambos métodos, aunque el ABP presenta algunas dificultades, como el tiempo que es necesario dedicar, tanto por profesorado como por alumnado (Wood, 2003), la dificultad en la transición a una nueva forma de aprender, así como las competencias necesarias del profesor para aplicarlos. 
BAENA-EXTREMERA, A. \& GRANERO-GALLEGOS, A. Concept mapping and problem-based learning content in anatomical and physiological in students for a public competition to be teachers in Secundary Education. Int. J. Morphol., 30(1):230-237, 2012.

SUMMARY: The aim of this study was to compare the learning achievement of students who prepared the opposition to be teachers in Secondary Education in the speciality of Physical Education, after receiving training in different thematic areas of anatomy and physiology through different teaching and learning methods: concept mapping (CMaps), problem-based learning (PBL) and combined use of both methods. Methodology: We used a quasi-experimental, descriptive and sectional methodological design. The total sample consisted of 180 students, 95 males and 85 females. We worked with a group of 64 people who received training through CMaps, another 61 students who received training PBL, and a third group of 55 which works by combining both methods. The measuring instrument was a questionnaire type assessment test for each content block. Pretest measure was obtained before training and other measures after the intervention (posttest). Improvements were analyzed in each group according to the method of teaching applied and compared the results obtained by analysis of gain scores (posttest-pretest) with T-test for related samples. Results: After intervention the mean score is above eighty percent in the five blocks analyzed, with the physiology and anatomy in the most evolved. PBL has proven more effective than CMaps, but the highest improvements, compared to pretest, are in the group that has studied combining CMaps + PBL. The results were discussed, in agreement with other authors, the need to consider greater use of PBL as a method of teaching and learning and using a mixed methodological approach students acquire greater skills and competencies.

KEY WORDS: Methodology; Concept mapping; Problem-based learning.

\section{REFERENCIAS BIBLIOGRÁFICAS}

Álvarez-Montero, S.; Caballero-Martin, M.A.; Gallego-Casado, P.; Nieto-Gómez, R.; Latorre de la Cruz, C. \& RodríguezMartínez, G. Sesiones basadas en escenarios clínicos: una aplicación del aprendizaje basado en la resolución de problemas. MEDIFAM, 11:83-91, 2001.

Alleyne, T.; Shirley, A.; Bennett, C.; Addae, J.; Walrond, E.; West, S. \& Pinto Pereira, L. Problem-based compared with traditional methods at the Faculty of Medical Sciences, University of the West Indies: a model study. Med. Teach., 24:273-9, 2002.

Ayala-Pimentel, J. O.; Díaz-Pérez, J. A. \& Orozco-Vargas, L. C. Eficacia de la utilización de estilos de aprendizaje en conjunto con mapas conceptuales y aprendizaje basado en la resolución de problemas para el aprendizaje de neuroanatomía. Educ. Méd., 12:25-31, 2003.

Best, J. W. Research in education. New Jersey, Prentice Hall, 1970.

Bollen, K. \& Long, J. S. Testing structural equation models. Newbury Park, Sage, 1994.

Canalejas, M.; Martínez, M.; Pineda, M.; Vera, M.; Soto, M.; Martín, A. \& Cid, M. Estilos de aprendizaje en los estudiantes de enfermería. Educ. Méd., 8:33-40, 2005.

Dueñas, V. H. El aprendizaje basado en problemas como enfoque pedagógico en la educación en salud. Colomb. Med., 32:18996, 2001 .

Edmondson, K. M. Concept Mapping for the Development of Medical Curricula. J. Res. Sci. Teach., 32:777-93, 1995.
Flórez-Uribe, A. M.; Ayala-Pimentel, J. O. \& Conde, C. C. Concept maps as a strategy for improving the teaching learning process in neuroanatomy. Int. J. Morphol., 29:84-9, 2011.

Fiedman, C.; Charles, P.; de Bliek, R.; Greer, D. S.; Mennin, S. P.; Norman, G. R.; Sheps, C. G.; Swanson, D. B. \& Woodward, C. A. Charting the winds of Change: Evaluating Innovative Medical Curricula. Acad. Med., 65:8-14, 1990.

Gal-Iglesias, B.; Busturia-Berrade, I. \& Garrido-Astray, M. C. Nuevas metodologías docentes aplicadas al estudio de la fisiología y la anatomía: estudio comparativo con el método tradicional. Educ. Méd., 12:117-24, 2009.

Gómez, F.; Rivas, I.; Mercado, F. \& Barjola, P. Aplicación interdisciplinar del aprendizaje basado en problemas (ABP) en ciencias de la salud: una herramienta útil para el desarrollo de competencias profesionales. RED U, 4: 2-19, 2009.

Hsu, L. L. Developing concept maps from problem-based learning scenario discussions. J. Adv. Nurs., 48:510-8, 2004.

Jones, A.; McArdle, P. J. \& O`Neill, P. A. Perceptions of how well graduates are prepared for the role of pre-registration house officer: a comparison of outcomes from a traditional and an integrated PBL curriculum. Med. Educ., 36:16-25, 2002.

Michael, J. In pursuit of meaningful learning. Adv. Physiol. Educ., 25:145-58, 2001.

Newman, A. The New Toronto Medical Curriculum. Bioch. Educ., 21:170-6, 1993. 
BAENA-EXTREMERA, A. \& GRANERO-GALLEGOS, A. Los mapas conceptuales y el aprendizaje basado en problemas en el aprendizaje de contenidos anatómico-fisiológicos en opositores al cuerpo de profesores de Educación Secundaria. Int. J. Morphol., 30(1):230-237, 2012.

Novak, J. D. Learning, Creating, and Using Knowledge: Concept Maps(R) as Facilitative Tools in Schools and Corporations. Mahwah, Lawrence Erlbaum Associates, 1998.

Novak, J. D. \& Gowin, D. B. Learning How to Learn. New York, Cambridge University Press, 1984.

Patel, V. L.; Groen, G. J. \& Norman, G. R. Effects of Conventional and Problem-based Medical Curricula on Problem Solving. Acad. Med., 66:380-9, 1991.

Rendas, A. B.; Fonseca, M. \& Pinto, P. R. Toward meaningful learning in undergraduate medical education using concept maps in PBL pathophysiology course. Adv. Physiol. Educ., 30:23-9, 2006.

Smits, P. B.; Verbeek, J. H. \& de Buisonjé, C. D. Problem based learning in continuing medical education: a review of controlled evaluation studies. BMJ, 324:153-6, 2002.

Soyibo, K. Using Concept Maps to Analyze Textbook Presentation of Respiration. Am. Biol. Teacher, 57(6): 344-51, 1995.

Williams, B. Case based learning--a review of the literature: is there scope for this educational paradigm in prehospital education? Emerg. Med. J., 22:577-81, 2005.

Wood, D. F. Problem based learning. BMJ, 326:328-30, 2003.

\author{
Dirección para correspondencia: \\ Dr. Antonio Baena Extremera \\ Facultad de Ciencias del Deporte. \\ C/ Argentina s/n 30720 \\ Santiago de la Rivera \\ Murcia \\ ESPAÑA
}

Email: abaenaextrem@um.es

Recibido : 18-07-2011

Aceptado: 02-11-2011 